J. Clin. Chem. Clin. Biochem.

Vol. 15, 1977, pp. 499-501

\title{
Jodbestimmung in Plasma und biologischen Flüssigkeiten im nmol/1-Bereich mittels Autoanalyzer
}

Von B. Glöbel

Aus dem Institut für Biophysik (Direktor: Prof. Dr. H. Muth) der Universität des Saarlandes Homburg (Saar)

(Eingegangen am 21. Februar/20. Mai 1977)

Zusammenfassung: Es wird eine automatische continuous-flow-Methode zur Bestimmung sehr geringer Konzentrationen von Jod in biologischen Flüssigkeiten angegeben. Sie hat eine Nachweisgrenze von $0,4 \mathrm{nmol} / \mathrm{l}$ Jodid und beruht auf dem analytischen Prinzip der Sandell-Kolthoff-Reaktion, wobei die Geschwindigkeit der Jodidkatalyse der Reduktion von $\mathrm{Ce}^{4+}$-Ionen durch Arsenit in schwefelsaurer Lösung gemessen wird.

Folgende Anwendungsbeispiele werden beschrieben:

Jodid im Plasma

Gesamtjod im Plasma

Proteingebundenes Jod

Gesamtjod im Urin
Gesamtjod im Trinkwasser

Jodgehalt von Rattenfutter

Jodgehalt von pflanzlichen Nahrungsmitteln

Gesamtjod in Kuhmilch

Determination of iodine in plasma and biological fluids in the range $2 \mathrm{nmol} / \mathrm{l}$ with the aid of an autoanalyser

Summary: An automated continuous flow method is described for the determination of iodine in biological fluids with a detection limit of $0.4 \mathrm{nmol} / \mathrm{l}$. Our method uses the kinetics of the Sandell-Kolthoff-Reaction in sulfuric acid for the determination of iodide. The following applications are described:

plasma inorganic iodine

total plasma iodine

protein bound iodine

total iodine in urine, drinking water and milk

total iodine in vegetables and laboratory diet for rats.

\section{Einführung}

Das analytische Prinzịp der Sandell-Kolthoff-Reaktion (1) beruht auf der Messung der Geschwindigkeit der jodidkatalysierten Reduktion von $\mathrm{Ce}^{4+}$ durch $\mathrm{As}^{3+}$ in schwefeḷaurer Lösung.

$2 \mathrm{Ce}^{4+}+\mathrm{As}^{3+} \longrightarrow 2 \mathrm{~J}^{-} \longrightarrow 2 \mathrm{Ce}^{3+}+\mathrm{As}^{5+}$

Im klinisch chemischen Laboratorium wird diese Reaktion in continuous flow Geräten zur Bestimmung des proteingebundenen Jod verwendet und hat eine untere Nachweisgrenze von etwa $80 \mathrm{nmol} / 1$. Die Jodidkonzentration im menschlichen Plasma hingegen liegt żwișchen $0,8-4.0 \mathrm{nmol} / 1$. Um derartig niedrige Konzentrationen an Jodid mit ausreichender Reproduzierbarkeit zu er: fassen, war es notwendig, die Empfindlichkeit der automatischen Methöde soweit zu steigern, daß mit ihr Konzentrationen bis hinab zu $0,4 \mathrm{nmol} / \mathrm{l}$ in beliebigen biologischen Flüssigkeiten bestimmint werden könnnen. Da viele organische Verbindungen die Sandell-Kolthoff-Reaktion beschleunigen oder hemmen, konnte auf die Veraschung nicht verzichtet werden. Der Einfluß von anorganischen Ionen außer Jodid ist in der Literatur ausreichend untersucht und muß bei jedem Meßproblem durch Zusatz steigender Mengen von Jodid zum Probenmaterial Berücksichtigung finden (1-9).

\section{Material und Methoden}

Abtrennung und Bestimmung des anorganischen Jod im Plasma

Das gesamte Verfahren ist in drei Schritte unterteilt:

1. Abtrennung des Jodid von Bestandteilen des Plasmas, die ein Molekulargewicht größer als 1000 besitzen (Ultrafiltration).

2. Nasse, saure, kontinuierliche Veraschung des Uiltrafiltrats.

3. Bestimmung der Jodidkonzentration durch Messung der Kinetik der Sandell-Kolthoff-Reaktion.

Zur Ultrafiltration des Plasmas mit $6 \mathrm{kPa}$ wurde die PelliconMembran PSAC von Millipore verwendet. 
Möglicherwveise vorhandenes freies Thyroxin und Trijodthyronin das normalerweise eine Konzentration von weniger als $0,3 \mathrm{nmol} / 1$ aufweist und durch die PSAC-Membran nur teilweise filtrierbar ist, kann bei der Bestimmung des Jodid vernachlässigt werden (9).

Die kontinuierliche Veraschung des Ultrafiltrats erfolgt in dem Technicon-Gerät $\mathrm{PB} / \mathrm{D} 1$ unter folgenden Bedingungen:

$\begin{array}{ll}\text { Temperatur: } & \text { Zone } 1220^{\circ} \mathrm{C} \\ & \text { Zone } 2230^{\circ} \mathrm{C} \\ \text { Umdrehungszahl: } & 3 \mathrm{U} / \mathrm{min} \\ \text { Chemikalien: } & \mathrm{H}_{2} \mathrm{SO}_{4} \text { p. a. } 970 \mathrm{~g} / \mathrm{kg} \\ & \mathrm{HNO}_{3} \text { p. a. } 650 \mathrm{~g} / \mathrm{kg} \\ & \mathrm{HClO}_{4} \text { p. a. } 650 \mathrm{~g} / \mathrm{kg}\end{array}$

Die Zusammensetzung des Veraschungsgemisches ist aus dem Fließschema zu entnehmen. Hierbei ist $\mathrm{HClO}_{4}$ mit $\mathrm{HNO}_{3}$ $80+20$ gemischt.

Am Ausgang des Veraschers erhält man etwa $990 \mathrm{~g} / \mathrm{kg} \mathrm{H}_{2} \mathrm{SO}_{4}$. Nach Verdünnung der Säure mittels $\mathrm{H}_{2} \mathrm{O}$ auf $450 \mathrm{~g} / \mathrm{kg}$ gelangt die Lösung in den analytischen Teil des Gerätes.

Die Kinetik der Sandell-Kolthoff-Reaktion ist bisher von zahlreichen Autoren (2-8) untersucht und zur Bestimmung von Jodid herangezogen worden. Dabei sind die optimalen Reaktionsbedingungen weitgehend festgelegt worden. Die Erfassungsgrenze einer kontinuierlich arbeitenden automatischen Anordnung hängt danach nur noch von der Empfindlichkeit und Stabilität des elektronischen Teils und der Gleichmäßigkeit des Reagenzflusses ab.

In Tabelle 1 sind die Bedingungen unserer Methode, die der Technicon-Methode zur Bestimmung von proteingebundenem Jod und die der Methode von Keller et al. (10) gegenübergestellt. Interferierende anorganische Substanzen wie $\mathrm{Cl}-\mathrm{F} ; \mathrm{Br}-\mathrm{SCN}$; $\mathrm{NH}_{4}{ }^{+}$und als Modellsubstanzen für organische Stoffe Tyrosin und Ascorbinsäure übten bis auf $\mathrm{Cl}^{-}$keinen meßbaren Einfluß auf das Bestimmungsverfahren aus. Die eingesetzten Konzentrationen entsprachen etwa dem Zehnfachen dessen, was „normalerweise" (11) maximal im Plasma erwartet werden kann.

\section{Verwendete Reagenzien}

Alle verwendeten Reagenzien, bis auf $\mathrm{As}_{2} \mathrm{O}_{3}$ und $\mathrm{NaJ}$, hatten den Reinheitsgrad p. a. und wurden von der Firma Merck, Darmstadt, bezogen. $\mathrm{As}_{2} \mathrm{O}_{3}$ war doppelt sublimiert und $\mathrm{NaJ}$ vom Reinheitsgrad Suprapur, beide ebenfalls von Firma Merck.

Zur Herstellung der Reagenzienlösungen wurde entmineralisiertes Wasser mit einem Jodgehalt von weniger als $0,1 \mathrm{nmol} / 1$ verwendet.

Die Ce ${ }^{4+}$ - und $\mathrm{As}^{3+}$-Lösungen haben wir in $0,5 \mathrm{~mol} / 1 \mathrm{H}_{2} \mathrm{SO}_{4}$ angesetzt, wobei die $\mathrm{As}^{3+}$-Lösung unter Erhitzen 4 Stunden mit einem Magnetrührer durchmischt wurde.

Abbildung 1 zeigt ein Fließschema der gesamten Anordnung. Das mitgeteilte Verfahren ist unter Umgehung der Ultrafiltration geeignet, Gesamtjod im Trinkwasser, verdünntem Urin (1:50) und Suspensionen von Nahrungsmitteln zu bestimmen. Auch das proteingebundene Jod kann nach Verdünnung des Plasmas und Entfernung des Jodid durch einen Ionenaustauscher direkt bestimmt werden. Suspensionen von pflanzlichen Nahrungsmitteln wurden durch Ultraschallzerkleinerung hergestellt. Dazu sind beliebige Laborquellen für Ultraschall verwendbar.

\section{Ergebnisse}

Bei 20-facher Bestimmung einer Einzelprobe erreichten wir mit $\bar{x} \pm s=0,438 \pm 0,032 \mathrm{nmol} / \mathrm{l}$ einen Variationskoeffizienten von $7,3 \%$.

In Tabelle 2 sind die in einigen Proben gemessenen Konzentrationen als Mittelwerte angegeben.

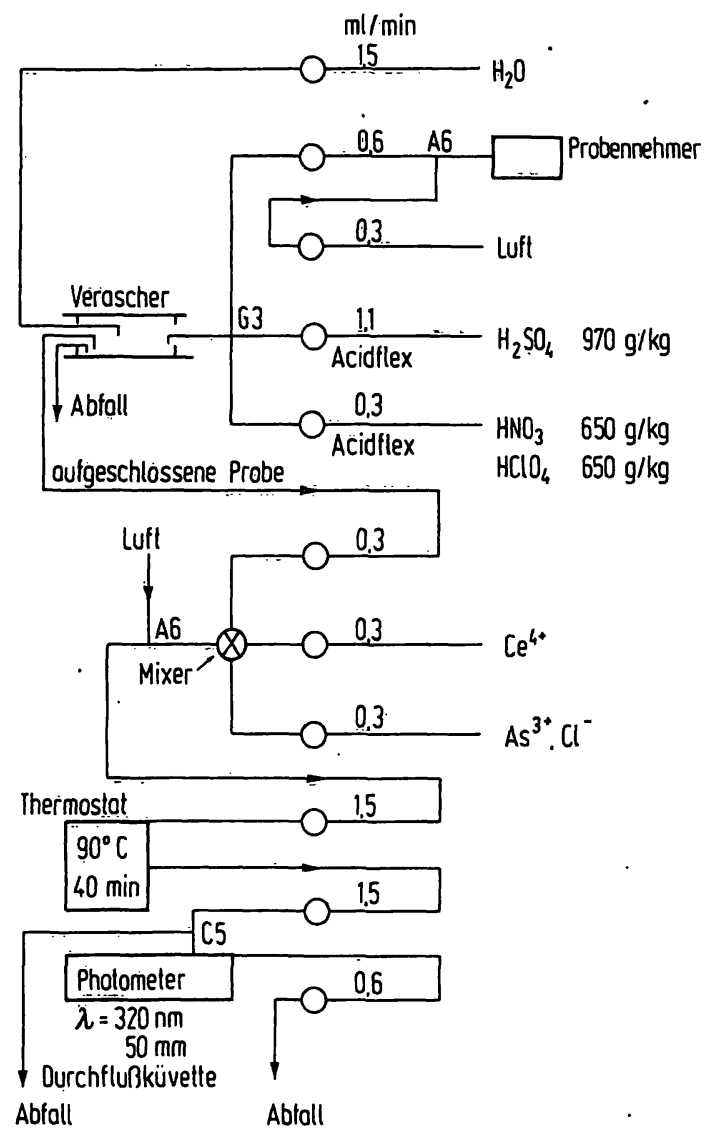

Abb. 1. Fließschema des continuous flow Autoanalysers

Tab. 1. Reaktionsbedingungen von drei Methoden zur Bestimmung des Jodid in Flüssigkeiten.

\begin{tabular}{|c|c|c|c|c|}
\hline & & proteingebundenes Jod & Keller et al. & eigene Methode \\
\hline Veraschung: & $\begin{array}{l}\text { Dauer [min] } \\
\text { Gemisch } \\
\text { Temperatur }\end{array}$ & $\begin{array}{l}\stackrel{3}{\mathrm{H}_{2} \mathrm{SO}_{4}, \mathrm{HNO}_{3}, \mathrm{HClO}_{4}} \\
50-280^{\circ} \mathrm{C}\end{array}$ & $\begin{array}{l}- \\
-\end{array}$ & $\begin{array}{l}10 \\
\mathrm{H}_{2} \mathrm{SO}_{4}, \mathrm{HNO}_{3}, \mathrm{HClO}_{4} \\
200-250^{\circ} \mathrm{C}\end{array}$ \\
\hline Analytik: & $\begin{array}{l}\mathrm{H}_{2} \mathrm{SO}_{4}[\mathrm{~mol} / 1] \\
\mathrm{Ce}^{4+}[\mathrm{mol} / 1] \\
\mathrm{As}^{3+}[\mathrm{mol} / 1] \\
\mathrm{As} / \mathrm{Ce} \\
\mathrm{NaCl}[\mathrm{mol} / 1] \\
t\left[{ }^{\circ} \mathrm{C}\right] \\
\mathrm{T}[\mathrm{min}]\end{array}$ & $\begin{array}{l}3,5 \\
0,002 \\
0,04 \\
20 \\
0,17 \\
55 \\
30\end{array}$ & $\begin{array}{l}0,37 \\
0,0017 \\
0,0065 \\
3,8 \\
0,34 \\
35 \\
35\end{array}$ & $\begin{array}{l}5 \\
0,0033 \\
0,033 \\
10 \\
0,01 \\
85-95 \\
40\end{array}$ \\
\hline Photometer: & $\begin{array}{l}\mathrm{nm} \\
\text { Schichttiefe } \mathrm{mm}\end{array}$ & $\begin{array}{r}415 \\
15\end{array}$ & $\begin{array}{r}420 \\
15\end{array}$ & $\begin{array}{r}320 \\
50\end{array}$ \\
\hline Nachweisgrenze: & $\mathrm{nmol} / 1$ & 80,0 & 1,6 & 0,4 \\
\hline
\end{tabular}


Tab. 2. Gemessene Mittelwerte und Bereiche bei einigen Probenmaterialien.

\begin{tabular}{|c|c|c|c|}
\hline Probe & Anzahl & $\begin{array}{l}\text { Mittel- } \\
\text { wert } \\
\text { [nmol/l] }\end{array}$ & $\begin{array}{l}\text { Bereich } \\
{[\mathrm{nmol} / 1]}\end{array}$ \\
\hline $\begin{array}{l}\text { Trinkwasser } \\
\text { Plasmajodid } \\
\text { Plasma-Gesamtjod } \\
\text { proteingebundenes Jod }\end{array}$ & $\begin{array}{r}30 \\
400 \\
20\end{array}$ & $\begin{array}{r}14 \\
8 \\
630\end{array}$ & $\begin{array}{c}3-28 \\
0,8-50 \\
236-1180\end{array}$ \\
\hline im Plasma & 60 & 530 & $150-1200$ \\
\hline Urinjod & 150 & 610 & $200-1800$ \\
\hline Jod in Kuhmilch & 40 & 250 & $60-2500$ \\
\hline Jod in Mineralwasser, Bier, Wein & 50 & 69 & $10-150$ \\
\hline Speichel, Mensch & 10 & 567 & $230-950$ \\
\hline Rattenfutter & 5 & 394 & $230-630$ \\
\hline Rattenfutter jodarm & 5 & 236 & $79-780$ \\
\hline Nahrungsmittel (pflanzlich) & 30 & 62 & $8-8000$ \\
\hline
\end{tabular}

\section{Diskussion}

Für proteingebundenes Jod im Plasma, Trinkwasser und Urin liegen die gemessenen Werte in dem in der Literatur angegebenen Bereich. Ein Vergleich der Jodidwerte im Plasma mit früheren Arbeiten ist nicht direkt möglich, da die bisher angegebenen Werte sehr unterschiedlich sind und mit anderen Methoden zum Teil auch indirekt bestimmt wurden $(11,12,13)$. Die von $E$. Klein angegebene Methode der direkten Bestimmung des Jodid ist durch die fehlende Veraschung des organischen Materials zu wenig spezifisch. So dürften die von ihm mitgeteilten relativ hohen Konzentrationen an Jodid im menschlichen Plasma auf den unkontrollierbaren Einfluß organischer Verbindungen in dem dort verwendeten Trichloressigsäureüberstand zurückzuführen sein. Die Bestimmung des anorganischen Jod im Plasma bei der Schilddrüsenfunktionsprüfung hat sich als sehr nützlich erwiesen. Insbesondere in Verbindung mit der Messung der Jodidclearance der Schilddrüse erhielten wir zuverlässige Werte für die absolute Jodidaufnahme der Schilddrüse (15-21); dies insbesondere dann, wenn andere Verfahren unklare oder keine Informationen über die Funktionslage des Organs erbrachten (22-28).

Für Jodbestimmungen im biologischen Bereich bringt das beschriebene Verfahren eine wesentliche Vereinfachung mit sich, da die Proben in großer Zahl auf automatischem Weg bestimmt werden und zudem nur minimalen Aufbereitungsaufwand erfordern. Alle angeführten Beispiele kommen ohne Anreicherung von Jod aus.

\section{Literatur}

1. Sandell, E. B. \& Kolthoff, I. M. (1973), Microchim. Acta 9-25.

2. Acland, J. D. (1957), Biochem. J. 66, 177-188.

3. Deman, J. (1964), Microchim. Acta 67-78.

4. Dubravcic, M. (1955), Analyst. 80, 295-300.

5. Kontaxis, N. E. \& Pickering, D. E. (1958), J. Clin. Endocrinol. Metab. 18, 774-786.

6. Lein, A. \& Schwartz, N. (1951), Anal. Chem. 23, 1507-1510.

7. Stolc, V. (1961), Microchim. Acta 710-720.

8. Strickland, R. D. \& Maloney, C.-M. (1957), Anal. Chem. 29, $1870-1873$.

9. Werner, S. C. \& Ingbar, S. H. (1971), The Thyroid, Third Edition, Harper \& Row, New York.

10. Keller, H. E., Doenecke, D. \& Leppla, W. (1968), Technicon Symposia 2, 371-376, Verlag Mediad Incorporated, White Plains, N. Y. 10601, U.S.A.

11. Documenta Geigy (1960), Wissenschaftliche Tabellen, 6. Aufl., Geigy J. R., A. G., Basel (Hrsg.).

12. Stelzer, M. \& Mertz, D. P. (1974), Therapiewoche, $820-825$.

13. Mertz, D. P. \& Stelzer, M. (1974), Therapiewoche, 2418-2424.

14. Klein, E. (1954), Biochem. Z. 326, 9-13.

15. Glöbel, B., Oberhausen, E. \& Berberich, R. (1972), Fortschr. Geb. Roentgenstr. Nuklearmed., Beiheft.

16. Oberhausen, E., Glöbel, B. \& Berberich, R. (1974), Nuklearmedizin, 156-158 (Pabst H. W., Hrsg.), F. K. SchattauerVerlag, Stuttgart.

17. Berberich, R., Glöbel, B. \& Oberhausen, E. (1974), Schilddrüse '73, 190-196, (Schleusener, H. \& Weinheimer, B., Hrsg.), G. Thieme Verlag, Stuttgart.

18. Berberich, R., Glöbel, B. \& Oberhausen, E. (1974), Comptes rendus du Colloque de Medicine Nucléaire de Langue Française 6.-9. 6. 1974 Clermont-Ferrand (Frankreich) 4, 73-82, Verlag Bloc Santé, Clermont-Ferrand.
19. Glöbel, B., Berberich, R. \& Oberhausen, E. (1974), Electromedica 3, 81-84, (Siemens Elema, Hrsg.) Verlag Druckhaus Nürnberg.

20. Berberich, R., Oberhausen, E., Glöbel, B., Debus, H. D. \& Probst, H. (1974), Electromedica 4, 111-114, (Siemens Elema Hrsg.) Verlag Druckhaus Nürnberg.

21. Glöbel, B., Berberich, R. \& Oberhausen, E. (1974), Nuc. Compact 5, 64-67, (Graul, E. H. \& Kuni, H., Hrsg.) GITVerlag, Darmstadt.

22. Glöbel, B., Oberhausen, E. \& Weinheimer, B. (1974), Nuc. Compact 2, 30-31, (Graul, E. H. \& Kuni, H. Hrsg.) GIT-Verlag, Darmstadt.

23. Mahlstedt, I., Schmidt, K., Glöbel, B. \& Joseph, K. (1975), Radioaktive Isotope in Klinik und Forschung 11, 496-502, (Höfer, R. Hrsg.) Urban und Schwarzenberg, München.

24. Mahlstedt, I., Schmidt, U., Joseph, K. \& Wenzel, K. W. (1974), Nuc. Compact 5, 50-53, (Graul, E. H. \& Kuni, H. Hrsg.) GIT-Verlag, Darmstadt.

25. Glöbel, B. (1975), Verhandlungsbericht der Schutzkommission beim Bundesminister des Innern, 237-239. Tagung Erlangen 1975 (Der Bundesminister des Innern, Hrsg.).

26. Berberich, R., Glöbel, B. \& Gotthier, R., Schilddrüse '75 Homburg (Saar), (Schleusener, H. \& Weinheimer, B. Hrsg.). G. Thieme Verlag, Stuttgart (im Druck).

27. Mahlstedt, J., Joseph, K., Schmidt, L., Meinhold, H., Wenzel, K.-W. \& Glöbel, B., Schilddrüse '75 - Homburg (Saar), (Schleusener, H. \& Weinheimer, B. Hrsg.) G. Thieme Verlag, Stuttgart (im Druck).

28. Glöbel, B., Oberhausen, E. \& Berberich, R. (1971). Nuc. Compact 6, 81-82 (Graul, E. H. \& Kuni, H. Hrsg.) GITVerlag, Darmstadt.

Dr. B. Glöbel Institut für Biophysik D-6650 Homburg/Saar 
